\title{
Utility of magnetic resonance imaging for differentiating malignant mesenchymal tumors of the uterus from $\mathrm{T} 2$-weighted hyperintense leiomyomas
}

\author{
Koichiro Matsuura $^{1}$ (D) Kaiji Inoue ${ }^{1} \cdot$ Eri Hoshino $^{1} \cdot$ Masanori Yasuda $^{2} \cdot$ Kosei Hasegawa $^{3} \cdot$ Yoshitaka Okada $^{4}$. \\ Yasutaka Baba ${ }^{4}$ Eito Kozawa ${ }^{1}$
}

Received: 18 June 2021 / Accepted: 28 October 2021 / Published online: 9 November 2021

(c) The Author(s) 2021

\begin{abstract}
Purpose To generate a new discrimination method to distinguish between malignant mesenchymal tumors of the uterus and T2-weighted hyperintense leiomyoma based on magnetic resonance imaging findings and clinical features.

Materials and methods Data from 32 tumors of 32 patients with malignant mesenchymal tumors of the uterus and from 34 tumors of 30 patients with T2-weighted hyperintense leiomyoma were analyzed. Clinical parameters, qualitative magnetic resonance imaging features, including computed diffusion-weighted imaging, and quantitative characteristics of magnetic resonance imaging of these two tumor types were compared. Predictive values for malignant mesenchymal tumors of the uterus were calculated using variant discriminant analysis.

Results The T1 bright area on qualitative assessment and mean apparent diffusion coefficient value on quantitative assessment yielded the most independent magnetic resonance imaging differentiators of malignant mesenchymal tumors of the uterus and T2-weighted hyperintense leiomyoma. The classification accuracy of the variant discriminant analysis based on three selected findings, i.e., a T1 bright area, computed diffusion-weighted imaging with a b-value of $2000 \mathrm{~s} / \mathrm{mm}^{2}(\mathrm{cDWI} 2000)$, and T2-hypointense bands, was $84.8 \%$ (56/66), indicating high accuracy.

Conclusions Variant discriminant analysis using the $\mathrm{T} 1$ bright area, $\mathrm{CDWI}_{2000}$, and T2-hypointense bands yielded high accuracy for differentiating between malignant mesenchymal tumors of the uterus and T2-weighted hyperintense leiomyoma.
\end{abstract}

Keywords Leiomyosarcoma $\cdot$ Endometrial stromal sarcoma $\cdot$ Undifferentiated uterine sarcoma $\cdot$ Leiomyoma $\cdot$ MRI

\section{Introduction}

Malignant mesenchymal tumors of the uterus (MMTUs), such as leiomyosarcoma, endometrial stromal sarcoma, undifferentiated uterine sarcoma, and smooth muscle tumor

Koichiro Matsuura

matsuurakoichiro0625@gmail.com

1 Department of Radiology, Saitama Medical University, 38, Morohongo, Moroyamamachi, Saitama, Japan

2 Department of Pathology, Saitama Medical University, 38, Morohongo, Moroyamamachi, Saitama, Japan

3 Department of Gynecologic Oncology, Saitama Medical University, 38, Morohongo, Moroyamamachi, Saitama, Japan

4 Department of Diagnostic Radiology, Saitama Medical University International Medical Center, 1397-1 Yamane, Hidaka, Saitama, Japan of uncertain malignant potential, are a rare heterogeneous group of malignant tumors of mesenchymal origin with poor prognosis [1-4]. Hysterectomy and bilateral oophorectomy are required for treating MMTUs [2-4]. In contrast, leiomyomas of benign mesenchymal tumors are the most common uterine neoplasms [1, 4-7]. For symptomatic leiomyoma treatment, minimally invasive surgery is needed to reduce postoperative pain, shorten hospitalization, and preserve fertility [4-7]. Therefore, accurate differentiation of malignant and benign mesenchymal tumors is critical for selecting the proper treatment.

Magnetic resonance imaging (MRI) is the most important diagnostic tool for differentiating between uterine leiomyoma and sarcoma [3, 8-18]. However, singleimage findings have limited distinguishing ability and are not considered sufficiently sensitive or specific, such as signs of hemorrhage, tumor margins, and apparent diffusion coefficient (ADC) values [3, 8-18]. Misdiagnosis or 
delayed diagnosis of MMTU is not uncommon in clinical practice, and one of the reasons is the similarity of MRI findings between MMTUs and uterine leiomyomas. For example, high signal intensity on T2-weighted images (T2WI) is one of the characteristics of MMTUs; however, uterine leiomyomas may also show hyperintensity due to degeneration, edema, and increased cellularity [19-21]. Therefore, it is difficult to distinguish between MMTUs and uterine leiomyomas that show a signal intensity higher than that indicated by the myometrium on T2WI, and it is clinically very important to differentiate them. Several studies have attempted to create a diagnostic method to distinguish between MMTUs and benign leiomyomas using multivariate analysis; however, they included low-signal leiomyomas on T2WI and had a limited sample size $[9,14,22]$. This study aimed to generate a new method to distinguish MMTUs and uterine leiomyomas wherein more than $50 \%$ of the lesions show higher signal intensity than that revealed by the myometrium on T2WI (T2-weighted hyperintense leiomyoma: T2HILM) based on the findings of MRI and clinical features.

\section{Materials and methods}

\section{Patient population}

Our Institutional Review Board approved this retrospective study, and informed consent was obtained in the form of an opt-out on the website.

One author with 30 years of experience retrospectively searched for eligible patients in our institutional pathological and MRI databases between May 2007 and August 2020. The inclusion and exclusion criteria were as follows: (1) patients with MMTU, cellular leiomyoma, and degenerative leiomyoma confirmed through pathology were included; (2) patients with tumors comprising more than $50 \%$ of areas of signal intensity higher than that of the myometrium on T2WI were included; (3) patients who underwent surgery and had complete MRI data within 3 months before surgery were included; (4) patients who were pregnant or with histories of preoperative chemotherapy, radiotherapy, or hormonal therapy were excluded; and (5) patients with carcinosarcoma were excluded according to the WHO Classification of Tumours. Female Genital Tumours, revised in the 5th edition [1].

The final study population consisted of 62 patients with surgically resected and pathologically proven MMTU (32 patients; 32 tumors; age range, 39-74 years; mean age, 56 years) or T2HILM (30 patients; 34 tumors; age range, 31-82 years; mean age, 49 years). The distribution of the pathological results is shown in Table 1.
Table 1 Pathological diagnosis

\begin{tabular}{ll}
\hline Pathologic results & $\begin{array}{l}\text { Number } \\
\text { of cases }\end{array}$ \\
\hline MMTU & 32 \\
Leiomyosarcoma & 16 \\
Endometrial stromal sarcoma (low grade) & 7 \\
Endometrial stromal sarcoma (high grade) & 5 \\
Undifferentiated uterine sarcoma & 2 \\
STUMP & 2 \\
T2HILM & 34 \\
Cellular leiomyoma & 15 \\
Hyaline degeneration & 4 \\
Hydropic degeneration & 2 \\
Mucinous degeneration & 1 \\
Cystic degeneration & 1 \\
Mixed degeneration & 11 \\
\hline
\end{tabular}

MMTU malignant mesenchymal tumors of uterus, STUMP smooth muscle tumor of uncertain malignant potential, T2HILM T2-weighted hyperintense leiomyoma

\section{Clinical parameters}

Information regarding age and abnormal vaginal bleeding was collected for patients with MMTU and T2HILM.

\section{MRI protocol}

Pelvic MRI was performed using a 1.5-T scanner (MAGNETOM Avanto 1.5 T, Siemens, Erlangen, Germany; or Nova Dual 1.5 T, Philips Healthcare, The Netherlands) and 3.0-T MR superconducting units (Intera Achieva 3.0 T; Philips Healthcare, Best, The Netherlands) with a phasedarray multicoil. T1WI and T2WI in the oblique sagittal section were obtained along the long axis of the uterus, and T2WI and diffusion-WI (DWI) in the transverse axial section were obtained. A three-dimensional fat-suppressed contrastenhanced T1WI protocol was included in the MRI. Before MRI, $20 \mathrm{mg}$ of butyl scopolamine (Buscopan, Boehringer Ingelheim, Ingelheim am Rhein, Germany) was intramuscularly injected to suppress bowel peristalsis. The MRI protocols are summarized in Table 2.

\section{Data processing and image interpretation}

ADC maps were generated automatically from each DWI $\left(b\right.$-values $=0,500$, and $\left.1000 \mathrm{~s} / \mathrm{mm}^{2}\right)$ using the MR system software. ADC histograms of every tumor slice were generated, and the mean ADC value, skewness, and kurtosis were calculated using commercially available software (SYNAPSE VINCENT version 4.4, Fujifilm, Tokyo, 
Table 2 Scan parameters of magnetic resonance imaging

\begin{tabular}{lllllllll}
\hline Parameters & T1WI & & T2WI & & FsGdT1WI & & DWI \\
& $1.5 \mathrm{~T}$ & $3.0 \mathrm{~T}$ & $1.5 \mathrm{~T}$ & $3.0 \mathrm{~T}$ & $1.5 \mathrm{~T}$ & $3.0 \mathrm{~T}$ & $1.5 \mathrm{~T}$ & $3.0 \mathrm{~T}$ \\
\hline TR $(\mathrm{ms})$ & $200-500$ & $150-600$ & $2500-5600$ & $3500-5600$ & $3-690$ & $3-700$ & $3200-6200$ & $6000-11,250$ \\
TE $(\mathrm{ms})$ & $2-12$ & $1-11$ & $80-105$ & $90-100$ & $1-12$ & $2-9$ & $70-80$ & $70-75$ \\
FOV $(\mathrm{cm})$ & $28 \times 32$ & $28 \times 33$ & $28 \times 35$ & $28 \times 36$ & $28 \times 38$ & $28 \times 39$ & $32 \times 35$ & $32 \times 36$ \\
Slice thickness $(\mathrm{mm})$ & $5-6$ & $4-5$ & $5-6$ & $4-5$ & $4-6$ & $2-5$ & $5-6$ \\
$b$ value $\left(\mathrm{mm}^{2} / \mathrm{s}\right)$ & - & - & - & - & - & - & $0,500,1000$ & $0,500,1000$ \\
\hline
\end{tabular}

T1WI T1-weighted image, T2WI T2-weighted image, FsGdT1WI post-contrast fat-saturated T1WI, DWI diffusion-weighted image, TR repetition time, $T E$ echo time, $F O V$ fields of view

Japan). Computed diffusion-weighted imaging (cDWIs) with $b$-values of $1500 \mathrm{~s} / \mathrm{mm}^{2}\left(\mathrm{cDWI}_{1500}\right), 2000 \mathrm{~s} / \mathrm{mm}^{2}\left(\mathrm{cDWI}_{2000}\right)$, $2500 \mathrm{~s} / \mathrm{mm}^{2}\left(\mathrm{cDWI}_{2500}\right), 3000 \mathrm{~s} / \mathrm{mm}^{2}\left(\mathrm{cDWI}_{3000}\right), 3500 \mathrm{~s} /$ $\mathrm{mm}^{2}\left(\mathrm{cDWI}_{3500}\right)$, and $4000 \mathrm{~s} / \mathrm{mm}^{2}\left(\mathrm{cDWI}_{4000}\right)$ were generated from real measured DWIs (rDWI) with $b$-values of 0 , 500 , and $1000 \mathrm{~s} / \mathrm{mm}^{2}$ by fitting a mono-exponential model using SYNAPSE VINCENT software.

Two radiologists with 6 and 4 years of experience, respectively, both blinded to the pathological diagnosis and clinical information, evaluated several MRI features for each index lesion. On qualitative assessment, any discrepancies were resolved by consensus after careful evaluation. Two readers independently assessed the following MRI features: main tumor location, presence of necrosis, cysts, T1 bright area, T2 dark areas, T2-hypointense band, feather-like enhancement, heterogeneity on T2WI, clarity of tumor margin (ill-defined or well-defined), tumor border shape (nodular or smooth), tumor morphology (round or amorphous), presence and location of unenhanced area (central or not), and signal intensity of tumor compared to the myometrium on DWI (rDWI and cDWI). Necrosis was defined as an irregular area with high signal intensity on T2WI and lack of enhancement after contrast medium administration (Fig. 1). Cysts were defined as well-demarcated areas without enhancement (Figs. 1 and 2). We defined a Tl bright area as a signal intensity that was higher or similar to that of the myometrium at pre-enhancement T1WI. It was defined as an indicator of subacute hemorrhage (Figs. 3 and 4). T2 dark areas represent areas of chronic hemorrhage that can be observed as a lower signal intensity area than that of the myometrium (Figs. 1 and 3). A T2-hypointense band was defined as the band-shaped low-signal area with equal signal to the
Fig. 1 A 76-year-old woman with endometrial stromal sarcoma without abnormal vaginal bleeding. a The axial T2-weighted image and $\mathbf{b}$ axial contrast-enhanced T1-weighted image with fat suppression show a tumor, located mainly in the myometrium, with hemorrhagic necrosis (arrow: necrosis and $\mathrm{T} 2$ dark area) and cyst (arrowhead). c On an axial diffusion-weighted image with a $b$-value of $1000 \mathrm{~s} /$ $\mathrm{mm}^{2}$ and $\mathbf{d}$ an axial computed diffusion-weighted image with a $b$-value of $2000 \mathrm{~s} / \mathrm{mm}^{2}$, the solid component of endometrial stromal sarcoma appears to have a higher signal than the myometrium (thin arrow). The lesion shows restricted diffusion, and the mean apparent diffusion coefficient value is $0.86 \times 10^{-3}$ $\mathrm{mm}^{2} / \mathrm{s}$
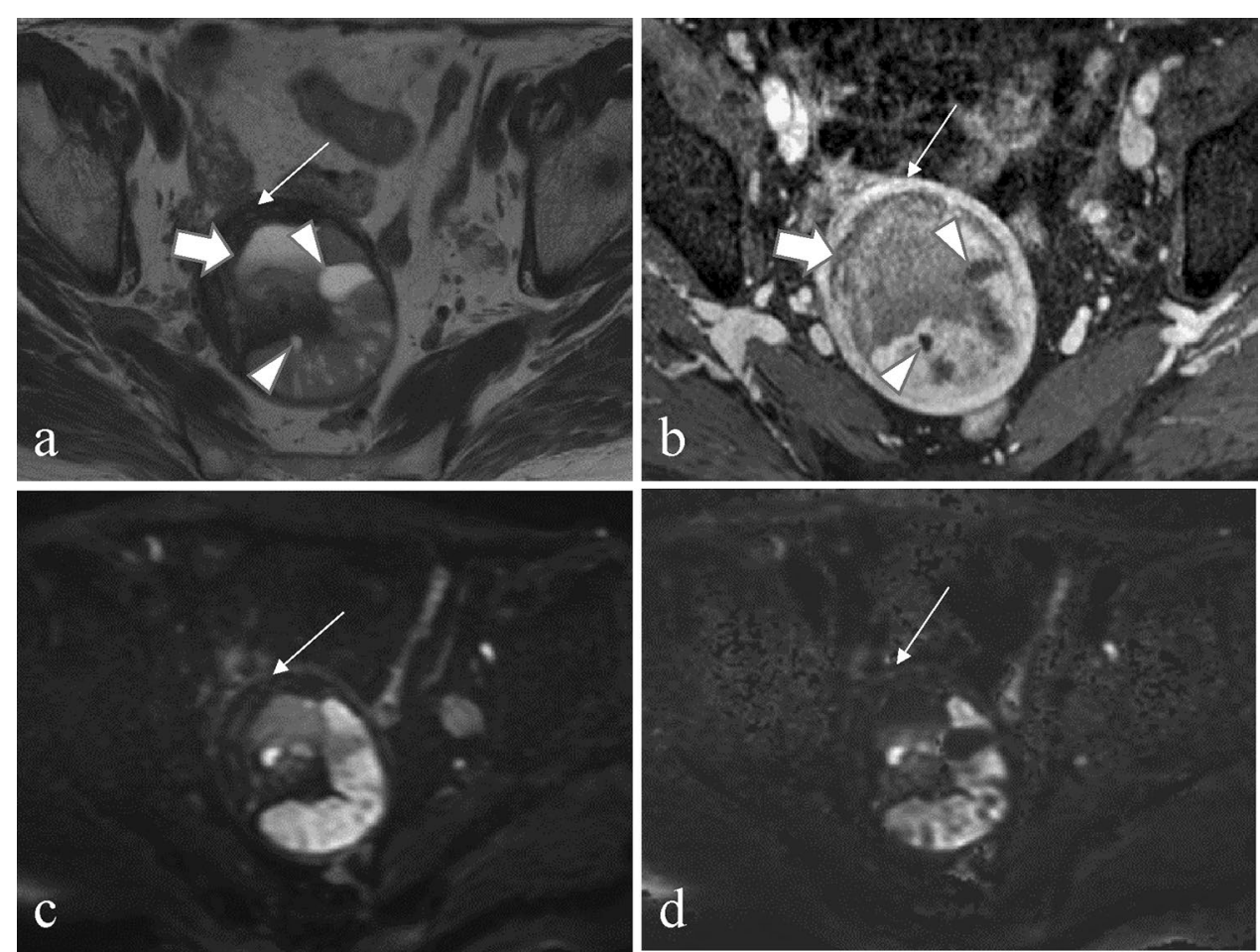
Fig. 2 A 48-year-old woman with endometrial stromal sarcoma without abnormal vaginal bleeding. a The sagittal T2-weighted image and $\mathbf{b}$ the sagittal contrast-enhanced T1-weighted image with fat suppression reveals a tumor, locates mainly in the myometrium, with cyst (arrowhead). a The sagittal T2-weighted image and $\mathbf{c}$ the axial $\mathrm{T} 2$-weighted image indicates the lesion with T2-hypointense bands (arrow) d On an axial diffusion-

weighted image with a $b$-value of $1000 \mathrm{~s} / \mathrm{mm}^{2}$, the tumor showed a higher signal than the myometrium (thin arrow). The lesion shows restricted diffusion, and the mean apparent diffusion coefficient value is $1.03 \times 10^{-3} \mathrm{~mm}^{2} / \mathrm{s}$
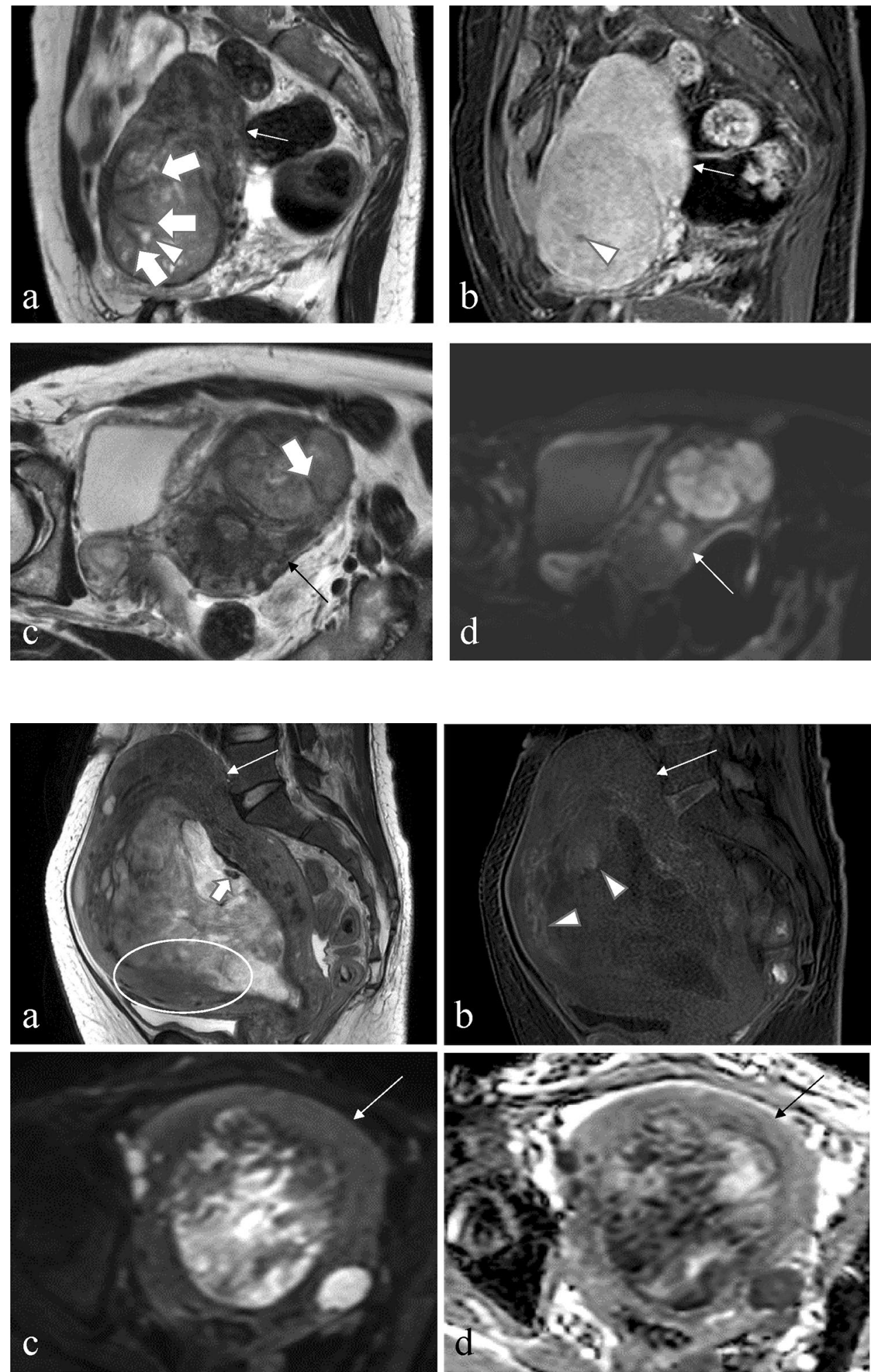

Fig. 3 A 56-year-old woman with leiomyosarcoma with abnormal vaginal bleeding. a The sagittal T2-weighted image shows a tumor, mainly located in the uterine cavity, with an ill-defined margin (circle) and T2 dark area (arrow). The tumor shows signal heterogeneity on the T2-weighted image. b The tumor shows a T1 bright area (arrowhead) on the sagittal T1-weighted image. c On an axial diffusion-weighted image with a $b$-value of $1000 \mathrm{~s} / \mathrm{mm}^{2}$, the lesion appears to have a higher signal than the myometrium (thin arrow). d The axial apparent diffusion coefficient map reveals that the solid component of leiomyosarcoma shows restricted diffusion, and the mean apparent diffusion coefficient value is $0.77 \times 10^{-3}$ $\mathrm{mm}^{2} / \mathrm{s}$ myometrium observed on T2WI within the lesion (Fig. 2). Feather-like enhancements were defined as fine and wispy enhancements interspersed within the tumors [23] as shown in Fig. 4. Ill-defined margins were defined as ambiguous and indistinguishable from the adjacent myometrium (Fig. 3).
DWI signals were evaluated as shown in Figs. 1, 2, 3, 4 and 5.

For quantitative assessments, two radiologists measured the data, and the data measured by the radiologist with 6 years of experience were used for the analysis. The ADC 
Fig. 4 A 49-year-old woman with undifferentiated uterine sarcoma with abnormal vaginal bleeding. a The sagittal T2-weighted image reveals a tumor located mainly in the uterine cavity, with a nodular border (circle). The tumor shows a heterogeneous signal on the T2-weighted image. b The tumor shows $\mathrm{T} 1 \mathrm{bright}$ area (arrowhead) on the sagittal T1-weighted image. $\mathbf{c}$ The contrast-enhanced T1-weighted image with fat suppression indicates a lesion with featherlike enhancement (rectangle). d On an axial diffusion-weighted image with a $b$-value of $1000 \mathrm{~s} /$ $\mathrm{mm}^{2}$, the solid component of undifferentiated uterine sarcoma shows a higher signal than the myometrium (thin arrow). The lesion shows restricted diffusion, and the mean apparent diffusion coefficient value is $0.90 \times 10^{-3} \mathrm{~mm}^{2} / \mathrm{s}$

Fig. 5 A 42-year-old woman with cellular leiomyoma without abnormal vaginal bleeding. a The coronal $\mathrm{T} 2$-weighted image reveals a tumor locates mainly in the subserosal location. The tumor shows a higher signal intensity than the myometrium on a $\mathrm{T} 2$-weighted image. b On a coronal diffusionweighted image with a $b$-value of $1000 \mathrm{~s} / \mathrm{mm}^{2}$, the lesion shows a higher signal than the myometrium (thin arrow). However, c the signal was equal to that of the myometrium on a coronal computed diffusion-weighted image with a $b$-value of 2000s/ $\mathrm{mm}^{2}$. d Using the coronal apparent diffusion coefficient map, the lesion is determined to have a mean apparent diffusion coefficient value of $1.65 \times 10^{-3}$ $\mathrm{mm}^{2} / \mathrm{s}$
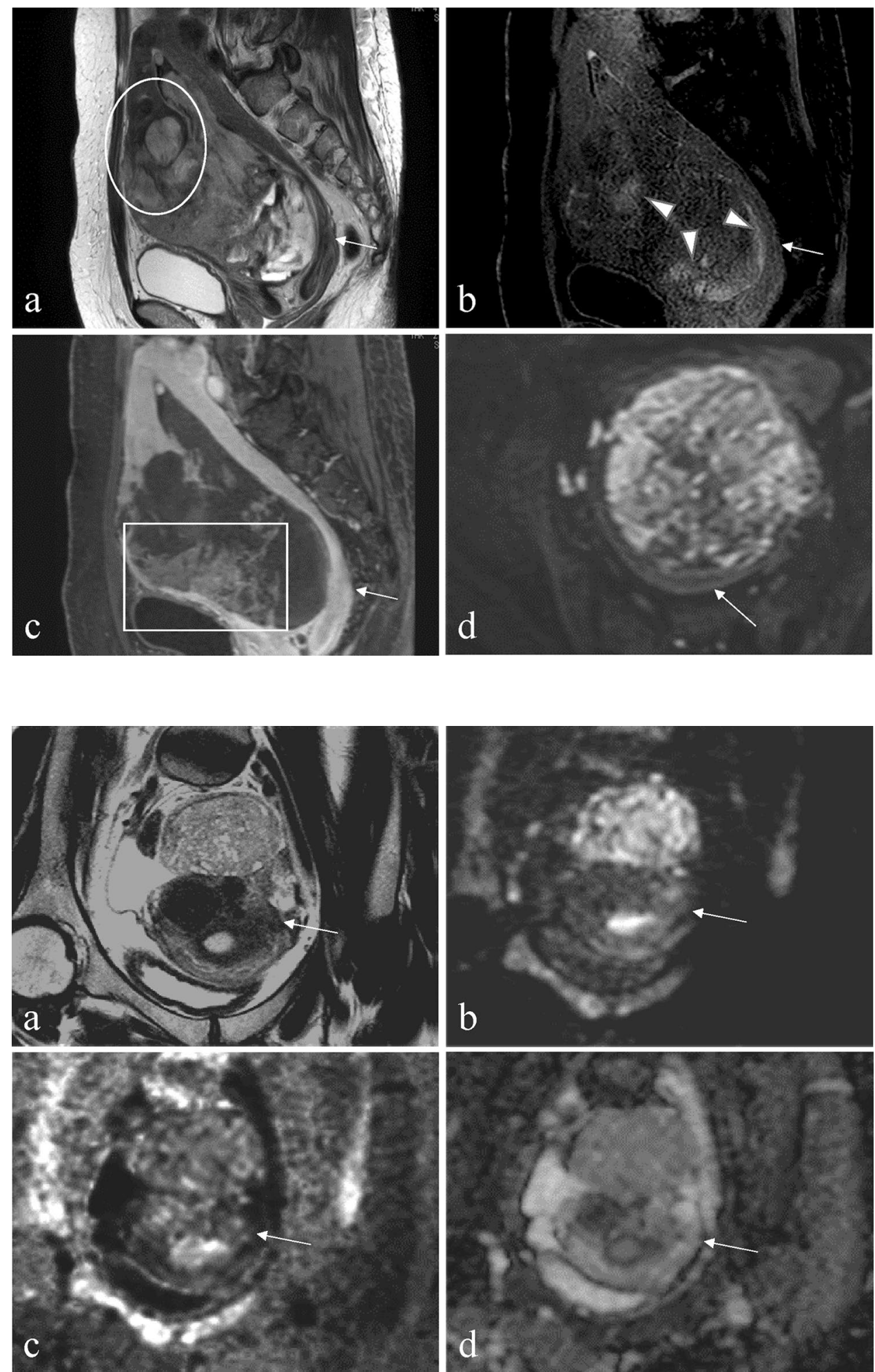

histogram values (mean ADC value, skewness, and kurtosis) of each primary tumor were measured using manually drawn regions of interest (ROI) on the console with solid components on the largest tumor plane, excluding the necrotic and non-enhanced portions by referring to T1WI, T2WI, and contrast-enhanced images. The largest diameter of the tumor and the non-enhancing area of the tumor (necrosis or cyst) was measured on transverse T2WI and fat-suppressed contrast-enhanced T1WI through the maximum face of the tumor. When there were multiple non-enhancing areas 
within the same tumor, the largest one was measured. Additionally, the maximum tumor area was measured.

\section{Statistical analysis}

We used several statistical tests to evaluate and analyze the capacity of visual and objective assessments to discriminate between MMTU and T2HILM. Statistical analyses were performed using JMP version 14.0.0 (SAS Institute; Cary, NC, USA). A $p$ value $<0.05$ was considered statistically significant. Qualitative interobserver agreement was calculated using the Kappa statistic; Kappa scores of $0.0-0.20,0.21-0.40,0.41-0.60,0.61-0.80$, and greater than 0.80 , indicated no-to-slight, fair, moderate, good, and excellent agreement, respectively [24]. Qualitative assessments between MMTU and T2HILM were analyzed using Fisher's exact test. Spearman's correlation coefficient and the intraclass correlation coefficient (ICC) were calculated to test the concordance of interobserver variability. The agreement was interpreted according to the ICC as follows: values less than $0.50,0.50-0.75,0.75-0.90$, and greater than 0.90 indicated poor, moderate, good, and excellent reliability, respectively [25]. We used the Wilcoxon test to compare the quantitative MRI characteristics. We performed receiver operating characteristic (ROC) curve analyses based on positive test results for patients with MMTU to identify the optimal cutoff for maximizing the sum of sensitivity and specificity. We examined the area under the ROC curve (AUC values of $0.5-0.7,0.7-0.9$, and $0.9-1.0$ refer to low, moderate, and high accuracy, respectively) [26] to assess the diagnostic abilities of the largest diameter of the tumor and the nonenhancing part of the tumor, maximum area of the tumor, mean ADC values, skewness, and kurtosis between MMTU and T2HILM.

A variant discriminant analysis was performed to gauge the associations of all qualitative and quantitative values using JMP version 14.0.0 and Multi Tahenryo version 1.1, an Excel add-in software (Institute of Statistical Analyses, Inc., Tokyo, Japan). Quantitative values were classified into two categories based on the AUC cutoff value for a variant discriminant analysis. If the discriminant accuracy rate is $75 \%$ or higher or the correlation ratio is 0.5 , the relationship equation can be applied to forecasting [27].

\section{Results}

\section{Qualitative MRI findings of MMTU and T2HILM}

The MRI findings of T2HILM differed significantly from those of MMTU, as summarized in Table 3. The interobserver agreement for MRI features was either good or excellent. Particularly, the agreement was excellent for the main location of the tumor component (0.99).

Only one of the T2HILMs demonstrated necrosis, a T2-hypointense band, and feather-like enhancement. In contrast, only one of the MMTUs demonstrated a lower signal than that of the myometrium on $\mathrm{rDWI}$ and $\mathrm{cDWI}_{1500}$, and one MMTU demonstrated well-defined margins. Necrosis, cysts, T1 bright area, T2-hypointense band, feather-like enhancement, clarity of tumor margin, tumor border shape, location of the unenhanced area, rDWI and $\mathrm{cDWI}_{1500-4000}$ showed significant differences $(p<0.05)$. The statistical analysis of each MRI finding is summarized in Table 4.

\section{Measurement of ADC maps in ROls}

The mean areas of the ROIs on ADC maps were $508.86 \mathrm{~mm}^{2}$ (range 103.13-2259.38 $\mathrm{mm}^{2}$ ) for MMTU and $939.08 \mathrm{~mm}^{2}$ (range 106.25-4462.77 $\mathrm{mm}^{2}$ ) for T2HILM. The ROI area for the histogram analysis of the ADC maps was significantly larger in T2HILM than in MMTU $(p=0.036)$.

\section{Quantitative MRI characteristics of MMTU and T2HILM}

In the comparison between MMTU and T2HILM, four MRI features, namely mean ADC value, skewness, kurtosis, and the largest diameter of the non-enhancing area, showed a significant difference $(p<0.05)$ (Table 5). ICC evaluation was excellent for tumor area and tumor diameter, moderate for the mean ADC value and diameter of the non-contrast area, and poor for skewness and kurtosis. The mean ADC values yielded moderate accuracy (AUC, 0.81; accuracy (ACC), 0.74 ) in differentiating between MMTU and T2HILM (Table 6). Similarly, the diameter of the non-enhancing area showed moderate accuracy (AUC, 0.77; ACC, 0.74), and skewness, kurtosis, tumor diameter, and tumor area showed low accuracy (Table 6).

The most discriminating mean ADC cutoff value to distinguish MMTU from T2HILM, as determined using ROC curve analysis, was $1.19 \times 10^{-3} \mathrm{~mm}^{2} / \mathrm{s}$. Similarly, the cutoff values for skewness, kurtosis, tumor diameter, non-contrast area diameter, area of the tumor, and age were $0.52,5.04$, $78.98 \mathrm{~mm}, 11.98 \mathrm{~mm}, 6166.50 \mathrm{~mm}^{2}$, and 55.00 years, respectively.

\section{Variant discriminant analysis}

The 19 qualitative MRI findings, six MRI characteristics, and two clinical parameters were set as independent variables (Tables 3 and 5). A variant discriminant analysis was performed. In total, three selected findings with the corresponding coefficients and the constants are presented in Table 7. The remaining 24 findings were excluded because 
Table 3 Qualitative MRI findings and a clinical parameter of T2-weighted hyperintense leiomyoma and MMTU

\begin{tabular}{lllll}
\hline MRI findings & $\begin{array}{l}\text { MMTU } \\
(n=32)\end{array}$ & $\begin{array}{l}\text { T2HILM } \\
(n=34)\end{array}$ & $\kappa$-value & $p$ value \\
\hline Main location of tumor: uterine cavity & $7(21.9 \%)$ & $4(11.8 \%)$ & 0.985 & 0.333 \\
Necrosis: present & $14(43.8 \%)$ & $1(2.9 \%)$ & 0.873 & $<.0001^{\dagger}$ \\
Cyst: present & $14(43.8 \%)$ & $5(14.7 \%)$ & 0.778 & $0.0139^{\dagger}$ \\
T1 bright area: present & $25(78.1 \%)$ & $5(14.7 \%)$ & 0.889 & $<.0001^{\dagger}$ \\
T2 dark area: present & $12(37.5 \%)$ & $6(17.6 \%)$ & 0.682 & 0.0983 \\
T2-hypointense band: present & $12(37.5 \%)$ & $1(2.9 \%)$ & 0.822 & $0.0004^{\dagger}$ \\
Feather-like enhancement: present & $16(50.0 \%)$ & $1(2.9 \%)$ & 0.855 & $<.0001^{\dagger}$ \\
Heterogeneity on T2WI: heterogeneous & $28(87.5 \%)$ & $29(85.3 \%)$ & 0.859 & 1.000 \\
Clarity of tumor margin: ill-defined & $31(96.9 \%)$ & $22(64.7 \%)$ & 0.680 & $0.0013^{\dagger}$ \\
Tumor border shape: nodular & $15(46.9 \%)$ & $2(5.9 \%)$ & 0.818 & $0.0002^{\dagger}$ \\
Tumor morphology: amorphous & $27(84.3 \%)$ & $25(73.5 \%)$ & 0.769 & 0.371 \\
Location of unenhanced area: central & $20(62.5 \%)$ & $4(11.8 \%)$ & 0.869 & $<.0001^{\dagger}$ \\
rDWI signal: higher than myometrium & $31(96.9 \%)$ & $25(73.5 \%)$ & 0.890 & $0.0134^{\dagger}$ \\
cDWI $_{1500}$ signal: higher than myometrium & $31(96.9 \%)$ & $23(67.6 \%)$ & 0.917 & $0.0029^{\dagger}$ \\
cDWI $_{2000}$ signal: higher than myometrium & $30(93.8 \%)$ & $18(52.9 \%)$ & 0.820 & $0.0002^{\dagger}$ \\
cDWI $_{2500}$ signal: higher than myometrium & $26(81.3 \%)$ & $15(44.1 \%)$ & 0.738 & $0.0024^{\dagger}$ \\
cDWI $_{3000}$ signal: higher than myometrium & $23(71.9 \%)$ & $8(23.5 \%)$ & 0.804 & $0.0002^{\dagger}$ \\
cDWI $_{3500}$ signal: higher than myometrium & $22(68.8 \%)$ & $6(17.6 \%)$ & 0.929 & $<.0001^{\dagger}$ \\
cDWI $_{4000}$ signal: higher than myometrium & $21(65.6 \%)$ & $6(17.6 \%)$ & 0.964 & $0.0001^{\dagger}$ \\
Abnormal vaginal bleeding: present $^{\dagger}$ & $10(31.3 \%)$ & $7(20.6 \%)$ & $\mathrm{NA}$ & 0.403 \\
\hline
\end{tabular}

MMTU malignant mesenchymal tumors of uterus, T2HILM T2-weighted hyperintense leiomyoma, T2WI T2-weighted image, $r D W I$ real measured diffusion-weighted image with $b$-value of $1000 \mathrm{~s} / \mathrm{mm}^{2}$, $c D W I_{1500-4000}$ computed diffusion-weighted image (with $b$-values of $1500-4000 \mathrm{~s} / \mathrm{mm}^{2}$ )

${ }^{\dagger}$ Significant difference between MMTU and T2-weighted hyperintense leiomyoma groups of low tolerance. A variable absent in the MRI findings, characteristics, and clinical parameters was set at 1 ; if it was present, the value was set at 2 , and substituted by the following formula:

$3.655 x_{1}+2.198 x_{2}+2.268 x_{3}-11.610$

where $x_{1}=\mathrm{T} 1$ bright area, $x_{2}=\mathrm{cDWI}_{2000}, x_{3}=\mathrm{T} 2$-hypointense band.

A negative discrimination score indicated T2HILM, and a positive discrimination score suggested MMTU. The classification accuracy of the variant discriminant analysis by combining the three selected findings of $\mathrm{T} 1$ bright area, cDWI $_{2000}$, and T2-hypointense bands was $84.8 \%$ or $56 / 66$ (T2HILM, 31/34; MMTU, 25/32), indicating high accuracy.

\section{Discussion}

MRI has a good diagnostic ability to differentiate between benign and malignant tumors. Multiple MRI findings were used to distinguish between MMTU and T2HILM. However, a single MRI finding, such as T1 bright area, necrosis, or feather-like enhancement, has limited distinguishing ability and demonstrates relatively low accuracy compared to the combination of multiple findings [14, 23, 28, 29]. As a quantitative assessment method, the usefulness of the mean ADC value and the maximum tumor size has been reported; however, there was an overlap between MMTU and leiomyoma $[8,18,23]$.

In our study, the presence of a T1 bright area, indicating subacute hemorrhage, had the highest accuracy (81.8\%) as an independent single MRI and clinical finding to significantly distinguish MMTU from T2HILM. The overall sensitivity, specificity, positive predictive value (PPV), and negative predictive value (NPV) of the T1 bright area were $78.1 \%, 85.3 \%, 83.3 \%$, and $89.6 \%$, respectively. However, in a previous report by Kim et al. [9], the accuracy, sensitivity, specificity, PPV, and NPV of hemorrhage were $58.7 \%$, $48.5 \%, 70.0 \%, 64.0 \%$, and $55.3 \%$, respectively, and there was no significant difference between MMTU and atypical leiomyoma. There are two possible reasons for this discrepancy. First, their MMTU included 55.5\% endometrial stromal sarcomas compared to $37.5 \%$ in our study. Their report, which included a large number of low-grade endometrial stromal sarcomas that did not cause substantial bleeding, may have shown a lower accuracy than that observed in this study, which had a large percentage of high-grade endometrial 
Table 4 Statistical analyses of qualitative MRI findings and a clinical parameter
Table 5 Quantitative MRI characteristics and a clinical parameter of $\mathrm{T} 2$ hyperintensity leiomyoma and MMTU

\begin{tabular}{llllll}
\hline MRI findings & ACC & Sensitivity & Specificity & PPV & NPV \\
\hline Main location of tumor & 0.561 & 0.219 & 0.111 & 0.179 & 0.138 \\
Necrosis & 0.712 & 0.438 & 0.971 & 0.933 & 0.647 \\
Cyst & 0.652 & 0.438 & 0.853 & 0.737 & 0.617 \\
T1 bright area & 0.818 & 0.781 & 0.853 & 0.833 & 0.896 \\
T2 dark area & 0.606 & 0.375 & 0.824 & 0.667 & 0.583 \\
T2-hypointense band & 0.682 & 0.375 & 0.971 & 0.923 & 0.623 \\
Feather-like enhancement & 0.742 & 0.500 & 0.971 & 0.941 & 0.673 \\
Heterogeneity on T2WI & 0.500 & 0.875 & 0.139 & 0.475 & 0.556 \\
Clarity of tumor margin & 0.659 & 0.969 & 0.353 & 0.585 & 0.923 \\
Tumor border shape & 0.712 & 0.469 & 0.941 & 0.882 & 0.653 \\
Tumor morphology & 0.545 & 0.844 & 0.265 & 0.519 & 0.643 \\
Location of unenhanced area & 0.758 & 0.625 & 0.882 & 0.833 & 0.714 \\
rDWI & 0.606 & 0.969 & 0.265 & 0.554 & 0.900 \\
cDWI $_{1500}$ & 0.636 & 0.969 & 0.324 & 0.574 & 0.917 \\
cDWI $_{2000}$ & 0.700 & 0.938 & 0.471 & 0.625 & 0.889 \\
cDWI $_{2500}$ & 0.682 & 0.813 & 0.559 & 0.634 & 0.760 \\
cDWI $_{3000}$ & 0.742 & 0.719 & 0.765 & 0.742 & 0.743 \\
cDWI $_{3500}$ & 0.758 & 0.688 & 0.824 & 0.786 & 0.737 \\
CDWI $_{4000}$ & 0.742 & 0.656 & 0.824 & 0.778 & 0.718 \\
Abnormal vaginal bleeding & 0.561 & 0.313 & 0.764 & 0.588 & 0.551 \\
\hline SC & & & & \\
\end{tabular}

$A C C$ accuracy, $P P V$ positive predictive value, $N P V$ negative predictive value, $T 2 W I$ T2-weighted image, $r D W I$ real measured diffusion-weighted image with $b$-value of $1000 \mathrm{~s} / \mathrm{mm}^{2}, c D W I_{1500-4000}$ computed diffusion-weighted image (with $b$-values of $1500-4000 \mathrm{~s} / \mathrm{mm}^{2}$ )

\begin{tabular}{lllll}
\hline $\begin{array}{l}\text { Characteristics } \\
(\text { Mean } \pm \mathrm{SD})\end{array}$ & $\begin{array}{l}\text { MMTU } \\
(n=32)\end{array}$ & $\begin{array}{l}\text { T2HILM } \\
(n=34)\end{array}$ & ICC & $p$ value \\
\hline Mean ADC value $\left(\times 10^{-3} \mathrm{~mm}^{2} / \mathrm{s}\right)$ & $1.029 \pm 0.208$ & $1.372 \pm 0.947$ & 0.688 & $<.0001^{\dagger}$ \\
Skewness & $0.758 \pm 0.634$ & $0.404 \pm 0.412$ & -0.0437 & $0.0078^{\dagger}$ \\
Kurtosis & $4.597 \pm 2.100$ & $3.450 \pm 0.911$ & 0.0758 & $0.0039^{\dagger}$ \\
Diameter of tumor $(\mathrm{mm})$ & $116.63 \pm 54.24$ & $90.89 \pm 53.96$ & 0.906 & 0.571 \\
Diameter of non-enhancing area $(\mathrm{mm})$ & $46.60 \pm 49.21$ & $11.39 \pm 28.28$ & 0.569 & $0.0004^{\dagger}$ \\
Area of tumor $\left(\mathrm{mm}^{2}\right)$ & $7409.32 \pm 4885.64$ & $5128.78 \pm 4618.92$ & 0.963 & 0.054 \\
Age (year) & $56.63 \pm 9.18$ & $49.85 \pm 10.23$ & $\mathrm{NA}$ & $0.006^{\dagger}$ \\
\hline
\end{tabular}

Values are given as mean \pm standard deviation about characteristics of MMTU and T2HILM

MMTU malignant mesenchymal tumors of uterus, T2HILM T2 hyperintensity leiomyoma, ICC interclass correlation coefficients

${ }^{\dagger}$ Significant difference between MMTU and T2 hyperintensity leiomyoma groups stromal sarcoma, leiomyosarcoma, and undifferentiated uterine sarcoma. Second, Kim et al. did not differentiate according to the time of bleeding, whereas we separately examined subacute and chronic hemorrhages. Malignant tumors are known to hemorrhage repeatedly within the tumor and more often than benign tumors. However, subacute bleeding may not be common in T2HILM. These reasons may have resulted in a relatively good accuracy in our study.

In our study, the ADC value of MMTU was significantly lower than that of T2HILM. The decreased ADC values could be attributed to the restricted motion of water molecules $[18,30]$. These results were similar to those of previous studies that reported lower ADC values in malignant sarcomas and endometrial stromal sarcomas than in benign leiomyomas [8, 18, 22, 23]. Furthermore, in this study, the ADC value showed the highest AUC (0.81) as a single quantitative parameter, which was slightly higher than the AUC of 0.74 reported for the combination of ADC value and DWI findings by Lin et al. [8], who compared the ADC values of leiomyosarcoma with those of ordinary leiomyoma. 
Table 6 Statistical analyses of quantitative MRI characteristics and a clinical parameter

\begin{tabular}{lllllllc}
\hline Characteristics & AUC & ACC & Sensitivity & Specificity & PPV & NPV & Cut off value \\
\hline Mean ADC value & $0.811^{\dagger}$ & 0.742 & 0.813 & 0.677 & 0.703 & 0.793 & 1.187 \\
Skewness & 0.650 & 0.636 & 0.563 & 0.706 & 0.643 & 0.632 & 0.520 \\
Kurtosis & 0.653 & 0.652 & 0.344 & 0.941 & 0.846 & 0.604 & 5.041 \\
Diameter of tumor & 0.642 & 0.636 & 0.750 & 0.529 & 0.600 & 0.692 & 78.98 \\
Diameter of non- & $0.766^{\dagger}$ & 0.742 & 0.719 & 0.824 & 0.742 & 0.743 & 11.98 \\
$\quad$ enhancing area & & & & & & & \\
Area of tumor & 0.661 & 0.667 & 0.594 & 0.735 & 0.679 & 0.658 & 6166.50 \\
Age & $0.705^{\dagger}$ & 0.667 & 0.594 & 0.794 & 0.826 & 0.596 & 55.00 \\
\hline
\end{tabular}

$A U C$ area under the receiver operating characteristic curve, $A C C$ accuracy, $P P V$ positive predictive value, $N P V$ negative predictive value

${ }^{\dagger}$ AUC as moderate accuracy
Table 7 Result of discriminant analysis

\begin{tabular}{lcll}
\hline & Discrimination factor & $F$ value & $p$ value \\
\hline T1 bright area & 3.655 & 21.30 & $<.0001$ \\
cDWI $_{2000}$ & 2.198 & 6.90 & 0.011 \\
T2-hypointense band & 2.268 & 5.93 & 0.018 \\
discriminate threshold & -11.610 & NA & NA \\
\hline
\end{tabular}

$c D W I_{2000}$ computed diffusion-weighted image (with $b$-values of $2000 \mathrm{~s} / \mathrm{mm}^{2}$ )

Ordinary leiomyomas show T2 blackout and yield low ADC values because of their fibrous components [18, 22]. We selected hyperintense leiomyomas on T2WI because malignant myometrial uterine tumors show high signal intensity. Therefore, our study showed a slightly higher AUC for differentiating between MMTU and T2HILM, although we only used mean ADC values.

Ordinary leiomyomas show signal homogeneity on T2WI [20], while MMTUs reveal signal heterogeneity [10]. Thomassin-Naggara et al. [22] showed that malignant uterine mesenchymal tumors were significantly more heterogeneous than benign leiomyomas, with accuracy, sensitivity, specificity, PPV, and NPV of $66.7 \%, 92.0 \%, 42.3 \%, 60.5 \%$, and $84.6 \%$, respectively. We expected that the heterogeneity on T2WI would be useful for distinguishing between MMTU and T2HILM; however, in our study, the accuracy, sensitivity, specificity, PPV, and NPV of heterogeneous signals were $50.0 \%, 87.5 \%, 13.9 \%, 47.5 \%$, and $55.6 \%$, respectively. Additionally, Lhakman et al. [14] used heterogeneity to differentiate between atypical leiomyomas and leiomyosarcomas; however, the accuracy (58.5\%) was not high. The sensitivity, specificity, PPV, and NPV were $94.7 \%, 27.3 \%, 52.9 \%$, and $85.7 \%$, respectively. Fifty-seven point seven percent and $22.7 \%$ of the leiomyomas included by Thomassin-Naggara et al. and Lhakman et al., respectively, were non-T2HILM ordinal leiomyomas, whereas we did not include these. Therefore, heterogeneity on T2WI may have become less significant for differentiation between T2HILM and MMTU in our study.

Qiu Bi et al. [28] reported that abnormal vaginal bleeding is a predictive factor for differentiating uterine sarcoma from atypical leiomyoma. However, in our study, the presence of abnormal vaginal bleeding was not a significant differentiating factor between MMTU and T2HILM as a single parameter and in the multivariate analysis. In the study by Qiu Bi et al., uterine sarcomas were located predominantly in the uterine cavity in approximately half of the cases, whereas in our study, most MMTUs were located in the myometrium. Moreover, the percentage of atypical leiomyomas in the uterine cavity was only $5 \%$, while the frequency of T2HILM cases in the uterine cavity was slightly higher at $12 \%$. There are many causes of abnormal vaginal bleeding, including disruption of vulnerable blood vessels associated with tumors [31]. The difference in the frequency of the main locus of the tumor may have altered the rate of abnormal vaginal bleeding due to vascular disruption.

In this study, the use of cDWI with a high $b$-value $\left(b=1500-4000 \mathrm{~s} / \mathrm{mm}^{2}\right)$ showed higher accuracy in differentiating MMTU from T2HILM than the use of rDWI. The detection and diagnosis of solid malignant components can be improved using a high b-value DWI in the prostate and breast regions [32-34]. Furthermore, in ovarian tumors, Takeuchi et al. [35] showed that visual evaluation of $\mathrm{cDWI}_{1500}$ could distinguish decidualized endometriomas from ovarian cancers. This is because the higher the $\mathrm{b}$-value, the greater the degree of signal attenuation from water molecules and the stronger the suppression of background normal tissues and other signals. However, the more cellular solid tumor area will continue to show a relatively high signal, accentuating the lesion [18]. In this study, there was no significant difference in discrimination ability when comparing b-values from 1500 to $4000 \mathrm{~s} / \mathrm{mm}^{2}$. An excessively high $b$-value leads to diminished visualization and the appearance of background normal tissues, resulting in excessive loss of the signals of necrosis, cystic degeneration, and 
tumor. Furthermore, the sensitivity and NPV decreased, and specificity and PPV increased, with an increasing b-value. Thus, there may be a limit to the improvement in diagnostic performance that can be achieved by increasing the b-value.

The rational application of variant discriminant analysis to integrate different image findings is a statistically valid and logical method for maximizing diagnostic accuracy. Some new findings and characteristics were added while referring to previous reports, and 27 findings and characteristics were used as variables in our study. Subsequent analysis of the contribution of variables to the results using the stepwise method eliminated 24 findings and characteristics and established a reasonable model. The combination of the three criteria, namely $\mathrm{T} 1$ bright area, a high signal at $\mathrm{CDWI}_{2000}$, and $\mathrm{T} 2$-hypointense band, was the indicator with the highest discrimination (84.8\%) for distinguishing MMTU from T2HILM.

Thomassin-Naggara et al. [22] found that the use of rDWI, mean ADC value, and T2 signal intensity in recursive partitioning model analysis achieved a diagnostic accuracy of $92.4 \%$ in distinguishing benign leiomyoma from MMTU. Qiu Bi et al. [28] proposed a model to differentiate uterine sarcoma from atypical leiomyoma, including T2HILM, using four features, e.g., abnormal genital bleeding, tumor located mainly in the uterine cavity, ill-defined tumor margins, and mean ADC value, and showed a high differentiation accuracy of $95.7 \%$. Their accuracy was attributable to analyzing features including rDWI, mean ADC value, and T2 signal intensity. Abnormal genital bleeding, tumor location being mainly in the uterine cavity, ill-defined tumor margins, and the mean ADC value showed slightly higher discrimination ability than our accuracy based on the $\mathrm{T} 1$ bright area, T2-hypointense band, and $\mathrm{cDWI}_{2000}$. However, in their recursive partitioning model, $\mathrm{T} 2$ hypointense tumors were selected as leiomyomas, whereas in our study, T2 hypointense leiomyomas or $\mathrm{T} 2$ isointense leiomyomas were excluded from the beginning because it was problematic for T2HILM to differentiate between a malignant and benign uterine myometrium. Our model has a sufficiently high and useful diagnostic capability compared to those previously reported.

Our study had several limitations. First, it was retrospective, and our exclusive inclusion of patients with surgically resected tumors and preoperative MRI may have introduced selection bias. Although it was necessary to ensure an accurate pathological diagnosis, prospective studies are needed to confirm our results. Second, our sample size was small; therefore, a large-scale multicenter study consortium is needed in the future. Third, our study included images from multiple scanners with both 1.5-T and 3.0-T MR systems to compare MMTU and T2HILM. However, the three MRI findings of our variant discriminant analysis contained only qualitative analysis factors and no quantitative factors.
However, our methods are widely applicable for diagnosing MMTU and T2HILM using both MR systems. Fourth, we did not compare the MRI findings with pathological findings. For example, T2 dark areas and T2-hypointense bands were distinguished from each other based on shape; however, the actual pathological findings were not confirmed. The imaging features of leiomyomas and MMTU identified in this study should be supported by radio-pathological correlation evaluations in future studies.

In conclusion, a T1 bright area on qualitative assessment and mean ADC value on quantitative assessment led to the most independent MRI differentiators of MMTU and T2HILM. Furthermore, variant discriminant analysis using the $\mathrm{T} 1$ bright area, $\mathrm{cDWI}_{2000}$, and $\mathrm{T} 2$-hypointense bands yielded high accuracy for differentiating between MMTU and T2HILM.

Acknowledgements We would like to thank Editage (www.editage. com) for English language editing.

\section{Declarations}

Conflict of interest We declare that we have no conflicts of interest with any organization or institute.

Ethical approval All procedures performed in studies involving human participants were in accordance with the ethical standards of the institutional and/or national research committee and with the 1964 Helsinki declaration and its later amendments or comparable ethical standards.

Informed consent Informed consent was obtained from all individual participants included in the study.

Open Access This article is licensed under a Creative Commons Attribution 4.0 International License, which permits use, sharing, adaptation, distribution and reproduction in any medium or format, as long as you give appropriate credit to the original author(s) and the source, provide a link to the Creative Commons licence, and indicate if changes were made. The images or other third party material in this article are included in the article's Creative Commons licence, unless indicated otherwise in a credit line to the material. If material is not included in the article's Creative Commons licence and your intended use is not permitted by statutory regulation or exceeds the permitted use, you will need to obtain permission directly from the copyright holder. To view a copy of this licence, visit http://creativecommons.org/licenses/by/4.0/.

\section{References}

1. Kim K-R, Lax SF, Lazar AJ, Longacre TA, Malpica A, MatiasGuiu X, et al. 6 Tumours of the uterine corpus. In: Cox J, editors. WHO classification of tumours. 5th ed. Female genital tumours. Lyon: International Agency for Research on Cancer; 2020. pp. 6 , 272-93.

2. D’Angelo E, Prat J. Uterine sarcomas: a review. Gynecol Oncol. 2010;116:131-9. 
3. Huang YT, Huang YL, Ng KK, Lin G. Current status of magnetic resonance imaging in patients with malignant uterine neoplasms: a review. Korean J Radiol. 2019;20(1):18-33.

4. Zhang J, Zhang J, Dai Y, Zhu L, Lang J, Leng J. Clinical characteristics and management experience of unexpected uterine sarcoma after myomectomy. Int J Gynaecol Obstet. 2015;130:195-9.

5. Stein K, Ascher-Walsh C. A comprehensive approach to the treatment of uterine leiomyomata. Mt Sinai J Med. 2009;76:546-56.

6. Schlaerth AC, Abu-Rustum NR. Role of minimally invasive surgery in gynecologic cancers. Oncologist. 2006;11:895-901.

7. Cagnacci A, Pirillo D, Malmusi S, Arangino S, Alessandrini C, Volpe A. Early outcome of myomectomy by laparotomy, minilaparotomy and laparoscopically assisted minilaparotomy. A randomized prospective study. Hum Reprod. 2003;18:2590-4.

8. Lin G, Yang LY, Huang YT, Ng KK, Ng SH, Ueng SH, et al. Comparison of the diagnostic accuracy of contrast-enhanced MRI and diffusion-weighted MRI in the differentiation between uterine leiomyosarcoma/smooth muscle tumor with uncertain malignant potential and benign leiomyoma. J Magn Reson Imaging. 2016;43:333-42.

9. Kim TH, Kim JW, Kim SY, Kim SH, Cho JY. What MRI features suspect malignant pure mesenchymal uterine tumors rather than uterine leiomyoma with cystic degeneration? J Gynecol Oncol. 2018;29(3):e26.

10. Santos P, Cunha TM. Uterine sarcomas: clinical presentation and MRI features. Diagn Interv Radiol. 2015;21:4-9.

11. Ohguri T, Aoki T, Watanabe H, Nakamura K, Nakata H, Matsuura $\mathrm{Y}$, et al. MRI findings including gadolinium-enhanced dynamic studies of malignant, mixed mesodermal tumors of the uterus: differentiation from endometrial carcinomas. Eur Radiol. 2002;12:2737-42.

12. Furukawa R, Akahane M, Yamada H, Kiryu S, Sato J, Komatsu $\mathrm{S}$, et al. Endometrial stromal sarcoma located in the myometrium with a low-intensity rim on T2-weighted images: report of three cases and literature review. J Magn Reson Imaging. 2010;31:975-9.

13. Cornfeld D, Israel G, Martel M, Weinreb J, Schwartz P, McCarthy S. MRI appearance of mesenchymal tumors of the uterus. Eur J Radiol. 2010;74:241-9.

14. Lakhman Y, Veeraraghavan H, Chaim J, Feier D, Goldman DA, Moskowitz CS, et al. Differentiation of uterine leiomyosarcoma from atypical leiomyoma: diagnostic accuracy of qualitative MR imaging features and feasibility of texture analysis. Eur Radiol. 2017;27(7):2903-15.

15. Koyama T, Togashi K, Konishi I, Kobayashi H, Ueda H, Kataoka ML, et al. MR imaging of endometrial stromal sarcoma: correlation with pathologic findings. AJR Am J Roentgenol. 1999;173:767-72.

16. Bharwani N, Newland A, Tunariu N, Babar S, Sahdev A, Rockall $\mathrm{AG}$, et al. MRI appearances of uterine malignant mixed mullerian tumors. AJR Am J Roentgenol. 2010;195:1268-75.

17. Teo SY, Babagbemi KT, Peters HE, Mortele KJ. Primary malignant mixed mullerian tumor of the uterus: findings on sonography, CT, and gadolinium-enhanced MRI. AJR Am J Roentgenol. 2008;191:278-83.

18. Tamai K, Koyama T, Saga T, Morisawa N, Fujimoto K, Mikami $\mathrm{Y}$, et al. The utility of diffusion-weighted MR imaging for differentiating uterine sarcomas from benign leiomyomas. Eur Radiol. 2008;18:723-30.

19. Ueda H, Togashi K, Konishi I, Kataoka ML, Koyamat, Fujiwara $\mathrm{T}$, et al. Unusual appearances of uterine leiomyomas: MR imaging findings and their histopathologic backgrounds. Radiographics. 1999;19:S131-45.
20. DeMulder D, Ascher SM. Uterine leiomyosarcoma: can MRI differentiate leiomyosarcoma from benign leiomyoma before treatment? AJR Am J Roentgenol. 2018;211(6):1405-15.

21. Yamashita Y, Torashima M, Takahashi M, Tanaka N, Katabuchi $\mathrm{H}$, Miyazaki K, et al. Hyperintense uterine leiomyoma at T2-weighted MR imaging: differentiation with dynamic enhanced MR. Radiology. 1993;189(3):721-5.

22. Thomassin-Naggara I, Dechoux S, Bonneau C, Morel A, Rouzier R, Carette MF, Darai E, Bazot M. How to differentiate benign from malignant myometrial tumours using MR imaging. Eur Radiol. 2013;23(8):2306-14.

23. Huang YL, Ueng SH, Chen K, Huang YT, Lu HY, Ng KK, et al. Utility of diffusion-weighted and contrast-enhanced magnetic resonance imaging in diagnosing and differentiating between high- and low-grade uterine endometrial stromal sarcoma. Cancer Imaging. 2019;19:63.

24. Landis JR, Koch GG. The measurement of observer agreement for categorical data. Biometrics. 1977;33:159-74.

25. Koo TK, Li MY. A guideline of selecting and reporting intraclass correlation coefficients for reliability research. J Chiropr Med. 2016;15(2):155-63.

26. DeLong ER, DeLong DM, Clarke-Pearson DL. Comparing the areas under two or more correlated receiver operating characteristic curves: a nonparametric approach. Biometrics. 1988;44:837-45.

27. Lachenbruch PA, Goldstein M. Discriminant analysis. Biometrics. 1979;35:69-85.

28. Bi Q, Xiao Z, Lv F, Liu Y, Zou C, Shen Y. Utility of clinical parameters and multiparametric MRI as predictive factors for differentiating uterine sarcoma from atypical leiomyoma. Acad Radiol. 2018;25:993-1002.

29. Xie H, Hu J, Zhang X, Ma S, Liu Y, Wang X. Preliminary utilization of radiomics in differentiating uterine sarcoma from atypical leiomyoma: comparison on diagnostic efficacy of MRI features and radiomic features. Eur J Radiol. 2019;115:39-45.

30. Colvin DG, Jourquin J, Xu J, Does MD, Estrade L, Gore JG. Effects of intracellular organelles on the apparent diffusion coefficient of water molecules in cultured human embryonic kidney cells. Magn Reson Med. 2011;65:796-801.

31. Livingstone M, Fraser IS. Mechanisms of abnormal uterine bleeding. Hum Reprod Update. 2002;8:60-7.

32. Blackledge MD, Leach MO, Collins DJ, Koh DM. Computed diffusion-weighted MR imaging may improve tumor detection. Radiology. 2011;261(2):573-81.

33. Metin V, Gökhan E, Aslıhan O, Ömer A, Tarık E, Yeşim S, et al. Conspicuity of peripheral zone prostate cancer on computed diffusion-weighted imaging: comparison of $\mathrm{cDWI}_{1500}, \mathrm{cDWI}_{2000}$, and cDWI $_{3000}$. Biomed Res Int. 2014;2014:768291.

34. Tamura T, Miyuki T, Higaki T, Yokomachi K, Akiyama Y, Sumida $\mathrm{H}$, et al. How to improve the conspicuity of breast tumors on computed high b-value diffusion-weighted imaging. Magn Reason Med Sci. 2019;18:119-25.

35. Takeuchi M, Kenji M, Harada M. Computed diffusion-weighted imaging for differentiating decidualized endometrioma from ovarian cancer. Eur J Radiol. 2016;85(5):1016-9.

Publisher's Note Springer Nature remains neutral with regard to jurisdictional claims in published maps and institutional affiliations. 